\title{
Estimation of Serum Electrolytes in Cases of Irritable Bowel Syndrome and Healthy Adults: A Comparative Study
}

\author{
Swathi Kamal ${ }^{1}$, Shubha Jayaram ${ }^{2}$, Sreenivas $\mathrm{N}^{3}$, Dharmendra $\mathrm{BL}^{4}$, Sudha B Sreenivas ${ }^{5}$
}

\begin{abstract}
Irritable bowel syndrome (IBS) is one of the most prevalent, multifactorial, heterogeneous and complex disorders that affects up to 1 in 5 people over their lifetime. It significantly reduces patients' quality of life. Individuals diagnosed with IBS have "low-grade intestinal inflammation", and increased intestinal permeability. This can create an electrolyte imbalance. This study was taken up as there is a paucity of literature and controversial reports on serum electrolytes in IBS.

Objectives: The objectives of this study were to estimate serum electrolyte levels in patients with IBS and to compare the same with that of normal healthy adults.

Materials and methods: This study was conducted on 108 individuals in the age group of 18-60 years of whom 54 were diagnosed IBS cases and 54 were normal controls. Estimation of serum electrolytes was done with the collected venous blood samples using the lon selective electrode technique in an electrolyte analyzer.

Results: Near normal mean serum sodium levels and a slight decrease in mean potassium level with a statistically significant increase in the mean serum chloride were the findings observed in our study group when compared with the controls.

Conclusion: IBS remains an enigmatic cause of significant distress, morbidity and disability. Our study implies that serum electrolyte levels are not greatly altered in IBS cases probably due to the rapid transit in colon, mucoidal consistency and small volume of stools. Lack of specific biomarkers adds to its complexity. Hence a multipronged approach is essential to improve the health related quality of life in these patients.

Clinical significance: Since this functional disorder is highly associated with significant emotional distress, impaired health-related quality of life, disability and high health care costs, this study tries to understand the possible changes that can be incorporated in the diet or serum electrolyte supplementation in IBS cases.
\end{abstract}

Keywords: Electrolyte analyzer, lon-selective electrode, Irritable bowel syndrome, Rome III criteria, Serum electrolytes.

Indian Journal of Medical Biochemistry (2019): 10.5005/jp-journals-10054-0082

\section{INTRODUCTION}

rritable bowel syndrome (IBS) is one of the most prevalent, multifactorial, heterogeneous and complex disorders that affects up to 1 in 5 people over their lifetime. It has a significant medical and socioeconomic impact that reduces patients' quality of life and it also imposes a significant economic burden to the healthcare system. ${ }^{1}$

The Rome III committee defines IBS as a chronic functional disorder characterized by recurrent abdominal pain or discomfort associated with disordered defecation [either constipation (IBS-C), diarrhea (IBS-D), or mixed/alternating symptoms of constipation and diarrhea (IBS-M), unidentified (IBS-U)].²

The pathophysiology of IBS is still poorly understood. It is most likely mediated by a combination of mechanisms including inflammation, autonomic dysfunction, dietary and psychological factors. The diagnosis and management of this disorder can be challenging. Several pathogenic factors responsible for IBS have been suggested, such as genetic and environmental factors, alterations in digestive motility, visceral hypersensitivity, inflammatory and post-infection mechanisms, psychological morbidity, and bacterial overgrowth among others. However, none of them seem to clearly explain the real mechanisms that trigger the syndrome. ${ }^{3}$

Individuals diagnosed with IBS have "low-grade intestinal inflammation", and increased intestinal permeability with changes in the intestinal microbiota. ${ }^{4}$ This can create an electrolyte imbalance. It is a well-known fact that maintaining normal serum electrolyte balance is essential for the efficient functioning of cells and organs in the body.

\begin{abstract}
${ }^{1}$ Student, ${ }^{2,3}$ Professor, ${ }^{4}$ Assistant Professor, ${ }^{5}$ Associate Professor
${ }^{1}$ Mysore Medical College and Research Institute, Mysuru, Karnataka, India

${ }^{2}$ Department of Biochemistry, Mysore Medical College and Research Institute, Mysuru, Karnataka, India

${ }^{3}$ Department of Pathology, Mysore Medical College and Research Institute, Mysuru, Karnataka, India

${ }^{4}$ Department of Surgery, Mysore Medical College and Research Institute, Mysuru, Karnataka, India

${ }^{5}$ Department of Physiology, JSS Academy of Higher Education and Research, Mysuru, Karnataka, India
\end{abstract}

Corresponding Author: Shubha Jayaram, Professor, Department of Biochemistry, Mysore Medical College and Research Institute, Mysuru, Karnataka, India, e-mail: drshubhajaysree@rediff.com

How to cite this article: Kamal S, Jayaram S, Sreenivas N, Dharmendra BL, Sreenivas SB. Estimation of Serum Electrolytes in Cases of Irritable Bowel Syndrome and Healthy Adults: A Comparative Study. Indian J Med Biochem 2019;23(1):185-188.

Source of support: Nil

Conflict of interest: None

Available studies regarding irritable bowel syndrome suggest controversial reports in the serum electrolyte levels. Some studies have shown that statistically significant improvement in abdominal discomfort or pain is observed when clinical trials with electrolyte supplementation were given. ${ }^{5}$

There is a paucity of literature directly linking the levels of the serum electrolytes and IBS, especially in south Indian studies. In

(O) The Author(s). 2019 Open Access This article is distributed under the terms of the Creative Commons Attribution 4.0 International License (https://creativecommons. org/licenses/by-nc/4.0/), which permits unrestricted use, distribution, and non-commercial reproduction in any medium, provided you give appropriate credit to the original author(s) and the source, provide a link to the Creative Commons license, and indicate if changes were made. The Creative Commons Public Domain Dedication waiver (http://creativecommons.org/publicdomain/zero/1.0/) applies to the data made available in this article, unless otherwise stated. 
addition, studies have suggested that the impact of IBS on healthrelated quality of life is equally as significant as in congestive heart failure and dialysis-dependent renal failure. Moreover, the association of the electrolyte levels with respect to the severity of the disease is not well established. Hence, the present study was taken up.

\section{Aims And OBJectives}

To measure the serum electrolyte levels in patients with IBS.

To compare the same with the serum electrolyte levels of normal healthy adults.

\section{Materials AND methods}

This case-control study was conducted in Mysuru at Mysore Medical College and Research Institute (MMC and RI), Mysuru, Karnataka, India on 108 individuals in the age group of 18-60 years of whom 54 were diagnosed IBS cases and 54 were normal controls. This age group was selected based on the facts about IBS by Intenational foundation for Gastro intestinal disorders and a study done on a large group of IBS patients who were between 18 years and 65 years of age. ${ }^{10}$ The sample size was calculated using standard deviation as 1.96 at $95 \%$ confidence level, the proportion of the population having the characteristic being measured as 0.07 , and sampling error of 0.05 . Institutional ethical clearance was obtained for this study.

\section{Inclusion Criteria}

Patients in the age group of 18-60 years diagnosed as IBS cases who fulfilled modified Rome III criteria for irritable bowel syndrome. i.e., recurrent abdominal pain or discomfort $\geq 3$ days per month in the last 3 months associated with $\geq 2$ of the following:

- Pain/discomfort improved after defecation.

- Onset associated with a change in the frequency of stool.

- Onset associated with a change in form (appearance) of stool, alternating between diarrhea and constipation.

- No evidence of any inflammatory, anatomical, a metabolic or neoplastic process that could cause the symptoms.

\section{Exclusion Criteria}

- Patients with known and established causes of diarrhea.

- Patients below 18 years and above 60 years.

- Patients who had diabetes mellitus, hypertension, a saltrestricted diet, steroid therapy, bronchial asthma, previous GI disorders, other systemic disorders.

- Patients on medications affecting serum sodium, potassium, and chloride levels.

- Procedure: Details were entered in a predesigned proforma that includes personal bio-data, presenting complaint, predominant symptoms, duration of illness and associated history relevant to exclusion criteria. Subjects were requested to fill up a detailed questionnaire (enclosed) related to bowel disorder.

Table1: Age distribution among cases and controls

\begin{tabular}{lll}
\hline Age (in years) & Controls & Cases \\
\hline$<30$ & 23 & 19 \\
$31-40$ & 7 & 15 \\
$41-50$ & 6 & 12 \\
$51-60$ & 18 & 8 \\
\hline
\end{tabular}

- Collection of blood sample: After obtaining informed consent under absolute aseptic precautions, $5 \mathrm{~mL}$ of venous blood samples were collected in red-capped vacutainers. Blood was allowed to clot and serum was separated by centrifugation of the sample at $5000 \mathrm{rpm}$ for 10 minutes and this sample was estimated for serum electrolytes-sodium, potassium and chloride ions using the direct ISE electrolyte analyzer (DIESTRO 103AP).

- Instrument used: Estimation of serum electrolytes was done by electrolyte analyzer. This high-throughput automated analyzer uses the principle of direct ion selective electrode (ISE) which is considered the best method for analysis of serum electrolytes. ISE is a special-purpose, potentiometric electrode consisting of a membrane, selectively permeable to a single ionic species. The potential produced at the membrane-sample solution interface is proportional to the logarithm of the ionic activity or concentration.

- Analysis of data: The collected data was analyzed using the following statistical methods. Descriptive statistics include mean, standard deviation, frequency, and percentage. The Descriptive procedure displays univariate summary statistics for several variables in a single table and calculates standardized values (z scores). Cramer's V test was employed in the present study for inferential statistics. The Independent-Samples T Test procedure compares means for two groups of cases. All the statistical methods were carried out through the SPSS for Windows (version 16.0).

\section{Observation and results}

The physical characteristics in this study include the mean age and the age distribution between the controls and cases. The mean age group of the controls and cases was 39 years and 36 years respectively. Out of 54 cases that formed the case study group, 19 were less than 30 years of age, 15 were in the age group of 31 to 40 years, 12 in the age group of 41-50 years and 8 were in the age group of 51-60 years (Table 1). A higher female preponderance was observed in this study (Table 2).

In comparison with the controls, the cases showed a difference of $1.17 \mathrm{mEq} / \mathrm{L}$ (i.e., a percentage decrease of 0.84 ) in their mean serum sodium levels. However, this fall was not statistically significant. Corresponding data is provided in Table 3 and represented in Graph 1.

Mean serum potassium levels also showed a similar trend like that of mean serum sodium levels in both groups. In comparison with the controls, the cases showed a fall of $0.18 \mathrm{mEq} / \mathrm{L}$ (i.e., a percentage decrease of 4.31) in their mean serum potassium levels. This fall was not statistically significant. Related data is provided in Table 3 and represented in Graph 2.

Interestingly, the mean serum chloride ions in cases indicated a rise of $2.31 \mathrm{mEq} / \mathrm{L}$ (i.e., a percentage increase of 2.27) when compared with that of the controls. This value was statistically significant. The data is provided in Table 3 and represented in Graph 3.

Table 2: Gender distribution in the study population

\begin{tabular}{lll}
\hline Gender & Cases & Controls \\
\hline Male & 20 & 30 \\
Female & 34 & 24 \\
\hline
\end{tabular}


Table 3: Comparison of mean serum electrolyte levels between cases and controls

\begin{tabular}{llllll}
\hline Mean \pm SD of Serum Electrolytes in $\mathrm{mEq} / \mathrm{L}$ & $N$ & Controls & Cases & $t$ value & 0.14 \\
\hline Sodium & 54 & $139.28 \pm 3.98$ & $138.11 \pm 4.19$ & 1.486 & 1.741 \\
Potassium & 54 & $4.35 \pm 0.51$ & $4.17 \pm 0.56$ & -1.956 & $0.05^{*}$ \\
Chloride & 54 & $101.11 \pm 6.90$ & $103.41 \pm 5.23$ & -1.956 \\
\hline
\end{tabular}

${ }^{*} p$ value $<0.05$ significant

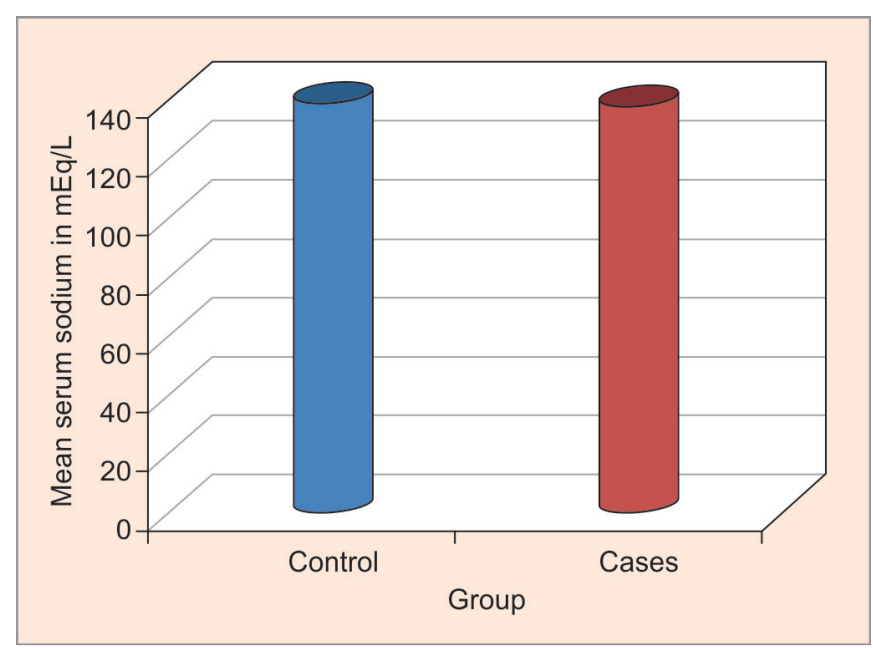

Graph 1: Comparison of mean serum sodium levels between cases and controls

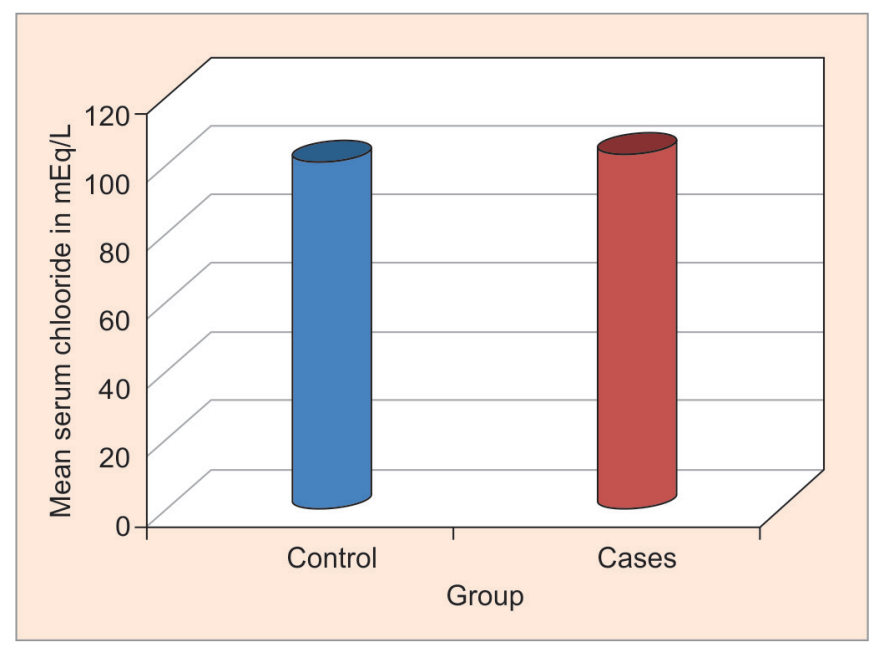

Graph 3: Comparison of mean serum chloride levels between cases and controls

Near normal mean serum sodium levels and a slight decrease in mean potassium level with a statistically significant increase in the mean serum chloride were the findings observed in our study group.

\section{Discussion}

IBS is a chronic functional symptom-based disorder and is characterized by multifactorial pathophysiological mechanisms including low-grade intestinal inflammation, ${ }^{4}$ autonomic dysfunction, hypersensitivity to diet and psychological distress. IBS lacks acceptable bio-markers, and its diagnosis largely depends on the exclusion of underlying organic disease. Hence the diagnosis and management of this disorder can be challenging.

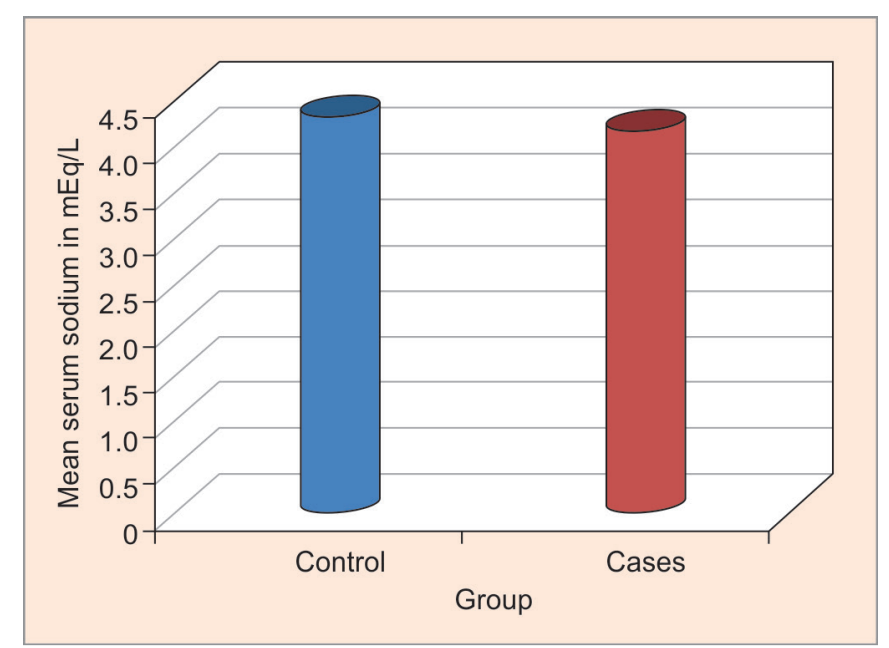

Graph 2: Comparison of mean serum potassium levels between the groups

Maintaining normal serum electrolyte balance is essential for the efficient functioning of living cells. One of the widely accepted views is that existing low-grade intestinal inflammation in IBS can cause increased intestinal permeability, which might possibly create an electrolyte imbalance.

Available studies regarding irritable bowel syndrome suggest controversial reports in the serum electrolyte levels. Some studies have shown that statistically significant improvement in abdominal discomfort or pain is observed when clinical trials with electrolyte supplementation were given. ${ }^{5}$

This study aimed to estimate the serum electrolyte values in IBS cases and the results suggested a reduction in serum sodium and potassium levels in these cases when compared with normal controls. Though there was a statistically significant increase in chloride levels in IBS cases, it was within the normal reference range of 98-108 mEq/L. The possible explanation for this increase could be due to defective chloride transporters on the intestinal mucosa.

The values obtained are in accordance with the study done on IBS diarrhea patients by Vernia et al., which also opines, serum electrolytes and systemic acid-base balance was within the normal range. These data are in agreement with the lack of systemic changes observed in IBS patients even with profuse or longstanding diarrhea. ${ }^{6}$

Although diarrhea is the predominant bowel dysfunction in as many as one-third of patients with IBS, it is unclear whether there is a specific disorder of intestinal fluid or electrolyte secretion in this syndrome, as there is no published evidence till date. ${ }^{7}$

Diarrhea in IBS is generally considered secondary to accelerated colonic transit and reduced volume of the proximal colon. ${ }^{8}$ The mucoid consistency and the small volume of the stools do not significantly affect the levels of serum electrolytes. This might be the probable reason for the lack of significant changes in the serum electrolyte levels in this study. 


\section{CONCLUSION}

Although a primary secretory diathesis has not been well documented in IBS, several mechanisms that could potentially contribute to intestinal secretion have been reported. Our study implies that serum electrolyte levels are not greatly altered in IBS cases probably due to the rapid transit in colon, mucoidal consistency and small volume of stools.

This functional disorder is highly associated with significant emotional distress, impaired health-related quality of life (HRQL), disability and high health care costs. ${ }^{9}$ The present study also tried to provide an insight into the possible changes that can be incorporated in the diet or serum electrolyte supplementation in IBS cases. Since the results were non-specific and statistically nonsignificant, further clinical studies are recommended with larger sample sizes for correlation of dyselectrolytemia with severity for obtaining conclusive evidence of the role of electrolyte supplements in the alleviation of both physical symptoms and psychological distress associated with this poorly understood disease.

\section{ACKNOWLedgments}

Authors are grateful to Dr Nandeesh HP, Dr Parveen Doddamani and Dr NK Shwetha of JSSAHER and Dr Lancy D'souza, statistics expert, for their timely help and support.

\section{References}

1. Lacy BE, Rosemore J, Robertson D, et al. Physicians'attitudes and practices in the evaluation and treatment of irritable bowel syndrome. Scand J Gastroenterol 2006;41:892-902.

2. Longstreth GF, Thompson WG, Chey WD, et al. Functional bowel disorders. Gastroenterology. 2006;130:1480-1491.

3. Pearson JS, Niven RM, Meng J, et al. Immunoglobulin E in irritable bowel syndrome: another target for treatment? A case report and literature review. Therap Adv Gastroenterol 2015;8:270-277.

4. Ortiz LM, Saz-Peiró P, Sebastián-Domingo JJ. Irritable bowel syndrome immune hypothesis. Part one: the role of lymphocytes and mast cells. Rev Esp Enferm Dig 2010;102:637-647.

5. Chapman RW, Stanghellini V, Geraint M, et al. Randomized clinical trial: macrogol/PEG 3350 plus electrolytes for treatment of patients with constipation associated with irritable bowel syndrome. Am J Gastroenterol 2013;108:1508-1515.

6. Vernia P, Latella G, Magliocca FM, et al. Seeking clues for a positive diagnosis of the irritable bowel syndrome. 1987;17(3):189-193.

7. Field M. Intestinal ion transport and the pathophysiology of diarrhea. J Clin Invest 2003;111:931-943.

8. Michael C. Intestinal Secretory Mechanisms in Irritable Bowel Syndrome-Diarrhea. Clin Gastroenterol Hepatol 2015;13(6):1051-1057.

9. Thompson WG, Longstreth GF, Drossman DA, et al. Functional bowel disorders and Functional abdominal pain in Rome II: functional gastrointestinal disorders: diagnosis, pathophysiology, and treatment. 2nd ed. Degnon Associates, Inc, McLean. VA; 2000: 351 432. 